Thorax (1954), 9, 299.

\title{
HEMIDIAPHRAGMATIC PARALYSIS AND PARESIS OF UNKNOWN AETIOLOGY WITHOUT ANY MARKED RISE IN LEVEL
}

\author{
BY \\ N. WYNN-WILLIAMS \\ From the Bedford General Hospital
}

(RECEIVED FOR PUBLICATION MAY 20, 1954)

Apart from eventration very little has been written about paralysis of the hemidiaphragm occurring without obvious cause. It is mentioned by Perry (1952), and Couch in 1953 reported 11 cases of paralysis of undetermined cause.

The purpose of this paper is to report 10 cases of paralysis of the hemidiaphragm and five cases of partial paralysis (paresis) for which no cause was found during the period of observation. All were associated with a small or moderate rise in the level of the affected dome. Three cases of eventration of the diaphragm and one of dissociated paresis due to prophylactic inoculation are also reported briefly to provide added material for discussing the relationship between the type of paralysis described here and eventration as well as its aetiology.

\section{Material AND CRITERIA}

All the cases described in this paper were seen among out-patients attending for the first time between the years 1948 and 1952 inclusive. These patients were referred because of symptoms, except for one who was discovered by mass radiography. Thirteen were seen at the Bedford Chest Clinic and two at the Luton Chest Clinic.

Some difficulty was found in defining the exact difference between paralysis and paresis of the diaphragm, as a few patients were seen who showed minimal descent of the diaphragm on quiet inspiration, but on deep inspiration showed immobility. In this paper immobility of the affected leaf of the diaphragm on deep, unhurried inspiration has been rated as paralysis. In every instance this was accompanied by paradoxical movement on sniffing. If definite but diminished movement downwards was present on deep inspiration, this has been termed paresis. Such paresis was accompanied by paradoxical movement on sniffing in two patients out of five. All radiographs were taken in the erect postero-anterior position at the end of a full but not forced inspiration.

Alteration in the level of the hemidiaphragm cannot be exactly judged even if a previous radiograph showing no abnormality in level is available. Observations on the films of over 100 normal men and women who had had serial radiographs taken showed that under chest clinic conditions the level of the diaphragm, as a whole, varied in nearly all from one film to another by up to $\frac{1}{2}$ in. $(1.3 \mathrm{~cm}$.). The usual difference in level between the two domes of the diaphragm in the same individual also varied occasionally by a similar distance, and appeared sometimes to be related to the stomach contents. It was found, however, that the commonest difference in height between the level of the right and left domes was $\frac{1}{2}$ in. $(1.3 \mathrm{~cm}$.) with a spread of from 1 in. $(2.5 \mathrm{~cm}$.) to zero, the right dome being the higher. For the purpose of this paper, therefore, the right dome has been estimated as being $\frac{1}{2}$ in. $(1.3 \mathrm{~cm}$.) higher than the left in every instance, and alterations in diaphragmatic height have been calculated on this arbitrary basis. Measurements to the nearest $\frac{1}{2}$ in. $(1.3 \mathrm{~cm}$.) have been taken by drawing a straight line through the vertebral column and extending further lines at right angles from the central line to the highest part of the domes of the diaphragm. Scoliosis, which would render this method inapplicable, was not met with in this series.

Periods under observation were equated to the nearest six months, but no case with less than one complete year under observation has been included.

\section{Paralysis of Hemidiaphragm}

Ten cases of paralysis of the hemidiaphragm, five right-sided and five left-sided, were observed during the years 1948 to 1952 . They occurred throughout the period; there were nine men and one woman. 
The youngest was aged 19 and the oldest 71 with an even spread between these extremes (Table 1).

Inquiry into the previous history of these patients disclosed no preceding illness that might be associated with the condition in eight. One patient, two months previously, had complained of pain in the neck on the same side as his paralysis. The pain was associated with headache and pyrexia, and the illness was severe enough to prevent work. The tenth patient stated that he was in the habit of drinking 20 pints of beer each day.

Symptoms included intermittent or chronic cough and sputum for many years in four; more recent cough and sputum up to one year in four. One had recurrent episodes of blood-spitting, five had dyspnoea on exertion, and two vague dyspepsia. Only two had been radiographed previously; the radiograph of one showed, two years before being seen, a similarly raised diaphragm, and the other a normal film. No evidence of organic nervous disease was found in any patient.

Pathological Investigations.-Examination of the sputum for tubercle bacilli proved negative in eight. The blood count was normal in six, the erythrocyte sedimentation rate was normal in seven, and the Wassermann reaction was negative in six. No other investigations were performed.

Radiological Findings.-The lung fields were normal in eight and showed old right apical inactive pulmonary tuberculosis on the opposite side to the paralysis in one. The tenth case had a calcified left hilar gland measuring $1 \mathrm{~cm}$. by $2 \mathrm{~cm}$. and left-sided diaphragmatic paralysis. It was presumed that there was no aetiological connexion, as this patient recovered half the normal movement of the affected leaf while under observation. Bronchograms done on three patients (including the one with haemoptysis) showed no abnormality.

When first seen the affected hemidiaphragm was paralysed and movement was paradoxical on sniffing in all. The paralysed dome was raised 2 in. $(5.1 \mathrm{~cm}$.) or less in eight out of the 10 cases, the remaining two, both on the left side, were raised $2 \frac{1}{2}$ in. $(6.3 \mathrm{~cm}$.) and $3 \frac{1}{2}$ in. $(8.9 \mathrm{~cm}$.) respectively. Details are shown in Table $\mathrm{I}$, and in this small series the rise was considerably more marked on the left than on the right side, averaging 1 in. $(2.5 \mathrm{~cm}$.) more.

Changes Noted during the Period of FollowUP.-Symptomatically there was little change during the observation period, which consisted of 33.5 observation years and averaged 3.4 years per patient; the longest was six years and the shortest one year (Table I).

The diaphragmatic movements showed no change in seven. In one half the normal movement returned with loss of paradoxical movement; movement had begun to return in one year and was maximal in three and a half years. A second case regained about one-thind movement in one and a half years and lost all paradoxical movement;

TABLE I

CLINICAL AND RADIOLOGICAL DATA ON 10 PATIENTS WITH PARALYSIS AND FIVE PATIENTS WITH PARESIS OF THE HEMIDIAPHRAGM

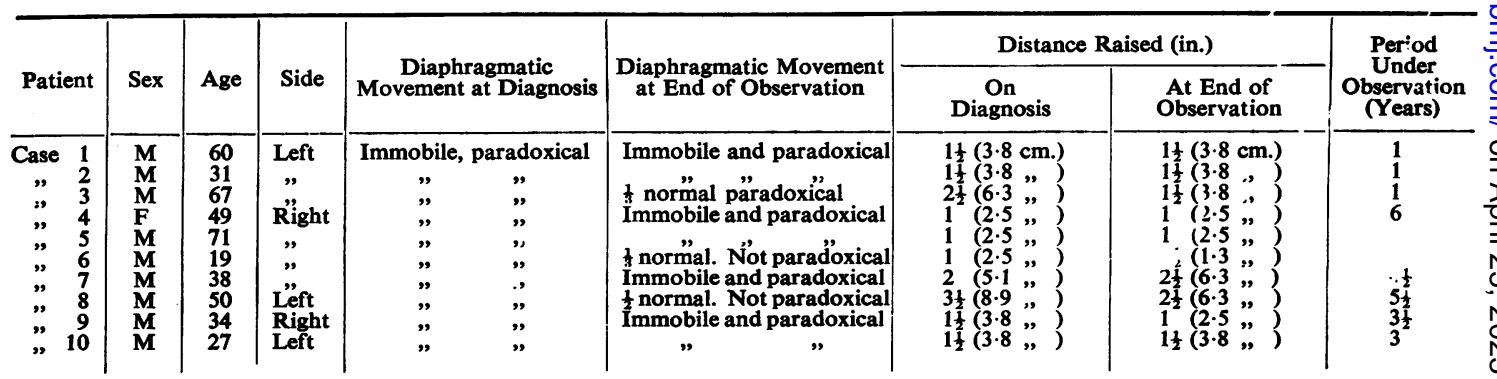

Patients with Paresis

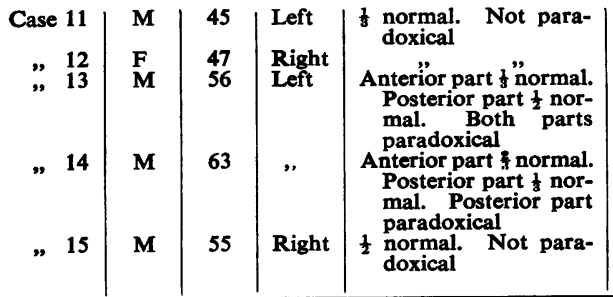

\begin{tabular}{|c|c|c|c|}
\hline t normal. Not paradoxical & $1(2.5 \mathrm{~cm})$. & $1 \frac{1}{2}(3.8 \mathrm{~cm})$. & $3 \frac{1}{2}$ \\
\hline $\begin{array}{l}\text { Anterior part" } 1 \text { normal. } \\
\text { Posterior part } \frac{1}{2} \text { normal. } \\
\text { Both parts paradoxical }\end{array}$ & $\begin{array}{l}1 \text { (2.5 ,, }) \\
\text { Anterior part } 1 \frac{1}{2} \\
(3.8 \mathrm{~cm} .) \\
\text { Posterior part } 2 \\
(5.1 \mathrm{~cm})\end{array}$ & $\begin{array}{l}1 \text { (2.5 ,, ) } \\
\text { Anterior part } 1 \frac{1}{2} \\
\text { (3.8 cm.) } \\
\text { Posterior part } 2 \\
\text { (5.1 cm.) }\end{array}$ & $\begin{array}{l}3 \frac{1}{2} \\
1\end{array}$ \\
\hline $\begin{array}{l}\text { Anterior part a normal } \\
\text { Posterior part } \frac{1}{2} \text { normal. } \\
\text { Posterior part para- } \\
\text { doxical } \\
\frac{8}{8} \text { normal. Not paradoxi- } \\
\text { cal }\end{array}$ & $\begin{array}{l}\text { Anterior part } 1 \\
(2.5 \mathrm{~cm} .) \\
\text { Posterior part } 2 \\
(5.1 \mathrm{~cm} .) \\
\frac{1}{2}(1.3 \mathrm{~cm} .)\end{array}$ & $\begin{array}{l}\text { Anterior part } 1 \\
(2.5 \mathrm{~cm} .) \\
\text { Posterior part } 2 \\
(5.1 \mathrm{~cm} .) \\
\frac{1}{2}(1.3 \mathrm{~cm} .)\end{array}$ & 41 \\
\hline
\end{tabular}


no further improvement occurred. The remaining case regained about one-third movement in one year (the length of observation), but paradoxical movements remained on sniffing.

The diaphragmatic level remained unaltered in five, and rose $\frac{1}{2}$ in. $(1.3 \mathrm{~cm}$.) in one. In the remaining patients the level of rise decreased by $\frac{1}{2}$ in. $(1.3 \mathrm{~cm}$.$) in three, and 1$ in. $(2.5 \mathrm{~cm}$.) in the remaining one, who was first seen with a rise of $3 \frac{1}{2}$ in. $(8.9 \mathrm{~cm}$.). The three patients who regained some movement all showed a decrease in height of the affected leaf.

\section{PARESIS OF Hemidiaphragm}

Five cases of paresis of the hemidiaphragm were seen, four male and one female; two were rightsided and three left-sided. One patient gave a history of pneumonia on the opposite side (unconfirmed radiologically) six weeks earlier. Accompanying symptoms and the results of pathological investigations were similar to those found in the patients with paralysis.

Radiological Findings.-The lung fields were clear in all. Diaphragmatic movement in two patients was about one-third of normal, in two between a half and one-third of normal, and in the fifth patient at least half normal. The affected leaf was raised 1 in. $(2.5 \mathrm{~cm}$.) in two patients, and $\frac{1}{2}$ in. $(1.3 \mathrm{~cm}$.) in one. The remaining two patients, both on the left side, showed the diaphragm raised and in two parts, the posterior part in each instance being the highest and moving least. Paradoxical movement was present in both sections in one patient and only in the posterior section in the other. The rise was $1 \frac{1}{2}$ in. $(3.8 \mathrm{~cm}$.) and 2 in. $(5.1 \mathrm{~cm}$.) in the first and 1 in. $(2.5 \mathrm{~cm}$.) and 2 in. $(5.1 \mathrm{~cm}$.) in the second. Details relating to each case may be seen in Table I, and it will be seen that the diaphragmatic level was higher on the left than on the right.

Changes Noted during the Period of FollowUP.- - The length of observation varied from 1 to 4.5 years. It consisted of 15.5 observation years and averaged 3.1 years per patient. In three of the five patients diaphragmatic movements and levels remained unaltered. In one movement increased during one year from about half to two-thirds of normal movement; the level remained the same. In the fifth patient movement was unaltered, but the diaphragmatic level rose $\frac{1}{2}$ in. $(1.3 \mathrm{~cm}$.).

\section{Hemidiaphragmatic Paralysis with Eventration}

Eventration of THE Diaphragm.-Three cases of typical eventration of the diaphragm were seen during the period. Two were in men and one in a woman. All were left-sided; the diaphragm was raised at least $3 \frac{1}{2}$ in. $(8.9 \mathrm{~cm}$.), immobile, and showed paradoxical movement on sniffing. One patient gave a history of injury to the left side of the chest with fractured ribs five years previously which had been followed by some dyspnoea on exertion. The second showed evidence of homolateral calcification of the pleura which may have been an aetiological factor. The third was typical of the idiopathic type of eventration in which there was no history of trauma or other causative factor and normal lung fields.

Paresis due to Serum Radiculitis.-Dissociated paresis of the left hemidiaphragm was seen in a man who had suffered from bilateral cervical radiculitis following an antitetanic serum inoculation. The condition of the diaphragm was similar to the two cases of dissociated paresis already described. There were residual signs of organic nervous disease in both arms.

\section{Discussion}

Hemidiaphragmatic paralysis due to frank interference with its nerve supply by trauma, neoplasm, or chronic pulmonary conditions such as tuberculosis has often been described. Less well known is diaphragmatic rise and immobility in association with abdominal and thoracic lesions. Inflammatory conditions in the abdomen may cause a high and immobile diaphragm (Harley, 1949; Meyler and Huizinga, 1950). Pneumonia (Freedman, 1950) and small pleural effusions (Meyler and Huizinga, 1950) may be accompanied by paradoxical movements on sniffing as well as immobility. Such immobility may be due to irritation of serous membranes causing relaxation with or without muscular spasm, but Meyler and Huizinga (1950) assumed that a true diaphragmitis secondary to the inflammatory process was the cause. A true neuritis of the phrenic nerve was thought responsible for paralysis arising with pneumonia by Freedman (1950). Joannides (1946) believed that a true primary diaphragmatis caused the immobility.

Paralysis due to these and allied causes is mentioned in order to exclude them as having any relation to the type of paralysis described in this paper, but eventration of the diaphragm must be considered fully, as it may well be an unmistakable example of the same condition. Eventration of the diaphragm is usually taken to mean a definite and marked elevation of the diaphragm. Königer (1909) suggested the term "idiopathic high-lying diaphragm," and stated that movement might be normal, diminished, absent, or reversed. Morison (1923a and b), Dillon (1928), and Kirklin and 
Hodgson (1947) reported a few cases in which the rise was less marked. The excellent review of the literature by Reed and Borden (1935) showed that the majority of cases described as eventration have had a markedly raised diaphragm, thought usually to be of congenital origin (aplasia), though a few cases have been described as acquired (atrophy). Lerche (1922) also accepted the possibility of an acquired origin and detailed many causes, such as typhoid, diphtheria, septicaemia, and alcoholic neuritis. Pacheco de Figueiredo and de Melo (1941) reported a case following lead poisoning. Quadrone (1912) mentioned influenza as a cause, and trauma, apart from that directed to the cervical plexus, has been blamed by Fatou, Prévost, and Prévost (1928). Eventration is commoner in men (4:1 Walton, 1924; 4: 3 Reed and Borden, 1935; 3:1 Kirklin and Hodgson, 1947; 7:1 Evans and Simpson, 1950). It is also commoner on the left than the right side $(59: 6$ Korns, $1921 ; 165: 18$ Reed and Borden, 1935; $30: 5$ Kinzer and Cook, 1944; 8:0 Evans and Simpson, 1950). Three cases of typical eventration are described in this paper, two of which fit into the acquired group and one into the idiopathic.

Paralysis of the hemidiaphragm without obvious cause appears to have been reported in the literature as eventration except by Couch (1953). He gave no details of the height of the paralysed dome, and of 11 cases eight were right-sided and six occurred in men. The ratio between the affected sides and, to a lesser extent, the sex-incidence were distinct from eventration.

The 15 cases of diaphragmatic paralysis and paresis reported in this paper showed certain differences from eventration, the most obvious of which was the extent of rise of the diaphragm, which was much less than in eventration. Also the right hemidiaphragm was affected as often as the left. On the other hand, the sex incidence of the palsies described in this paper was not unlike that in eventration, and the thinness of the affected diaphragm, whenever outlined by air in the stomach or bowel, was like that seen in eventration. Moreover, the greater average rise of the left hemidiaphragm should be noted. Consideration of these facts suggests the possibility that eventration of the diaphragm is essentially similar to the type of paralysis described in this paper, the preponderance of left-sided cases of eventration reported being due to the fact that the left hemidiaphragm rises higher than the right when paralysed and that only cases with a marked rise of the hemidiaphragm have been described except rarely. Paresis is found when some degree of return of movement occurs after paralysis. This sequence was observed in three cases out of 10 in this series. It is also likely that paresis of the hemidiaphragm may occur without complete paralysis, as is observed occasionally after crushing the phrenic nerve.

The patients with diaphragmatic paresis showed no essential difference from those with paralysis, but in two of the five cases there was dissociated paresis of the anterior and posterior parts of the left hemidiaphragm. I have been unable to trace any reference to such paresis, but Couch (1953) described dissociated paralysis of the right diaphragm (one case of undetermined cause). Earlier Hitzenberger (1927) reported paralysis of the anterior part of the right diaphragm and normal movement of the posterior part caused by syphilitic cerebrospinal meningitis, and Abeles and Leiner (1944) recorded a similar case following poliomyelitis. Douady, Lardanchet, and Venator (1939, two cases) and Fox (1948, one case) noted dissociated paralysis of the right hemidiaphragm following phrenic crush and pneumoperitoneum. They showed that dissociated paralysis might only be seen after the induction of pneumoperitoneum. Certainly the frequent presence of air under the left diaphragm might be expected to render the condition more obvious on this side. The two cases of dissociated paresis of unknown cause and the one following cervical radiculitis described in this paper were all left-sided, and the striking appearance of two arcs outlined by air led at once to screening.

The case histories of the 15 patients throw very little light on any aetiological cause for the paralysis. One patient gave a history of previous pain in the neck on the side of the paralysis, and another of the excessive intake of alcohol. In both of these it is possible that an isolated neuritis of the phrenic nerve occurred, but this would be atypical of $ᄋ$ neuritis due to alcohol. It has been suggested by Shanks and Kerley (1951) that subclinical polio- 은 myelitis might be a cause, but again it seems unlikely $\frac{D}{0}$ that the nuclei of the phrenic nerve would often be picked out alone and no other group of muscles $N$ damaged. Abeles and Leiner (1944), reporting $N$ this condition for the first time, described it in company with widespread skeletal paralysis. Iso- $\omega$ lated neuritis due to virus or other infections has? occasionally been recorded (Richardson, 1942), but $\stackrel{\circ}{\complement}$ never affecting the phrenic nerve alone. Such a theory of causation might account for a small $\stackrel{+}{+}^{+}$ number of cases. Others might also be due to the $\frac{0}{0}$ paralysis which may accompany pneumonia remain- $\frac{}{\mathbb{D}}$ ing permanent; for pneumonia is very common $\frac{?}{\mathbb{D}}$ and many cases are never radiographed, and even $\varrho$ if radiographed seldom screened to study the? 
diaphragmatic movement. Similar remarks apply to the hemidiaphragmatic paralysis that may follow pulmonary infarction, recently described by Macleod and Grant (1954).

The case of dissociated paresis of the hemidiaphragm following antitetanic serum was reported because of its possible aetiological bearing. Miller and Stanton (1954) have reported neurological sequelae following prophylactic inoculations of many kinds. Isolated paralysis of one muscle has been described after such inoculations, but not of the hemidiaphragm alone, although in company with other muscles it has been affected (French, 1938). In view of the immense number of such injections that have been given, particularly to men, it remains a remote possibility. No helpful information was obtained in this investigation, except that one or more injections of uncertain composition had been given at some period to the majority of the 15 patients. In the absence of more knowledge further speculation appears unprofitable.

\section{SUMMARY}

Ten cases of paralysis and five of paresis of the hemidiaphragm without marked rise in level of the affected dome and of unknown aetiology are reported. Thirteen occurred in men and two in women; the right side was affected in seven and the left in eight; the average degree of rise was more marked on the left side than on the right.
Reasons are put forward for thinking that there is no essential difference between this type of paralysis and eventration of the diaphragm. Possible aetiological causes are discussed, but no satisfactory conclusions emerged to explain this comparatively common palsy.

I am indebted to Dr. J. Brian Shaw for the clinical and radiological details of Cases 9 and 10 .

\section{REFERENCES}

Abeles, H., and Leiner, G. C. (1944). Amer, J. Roentgenol., 51, 572 Couch, A. H. C. (1953). Thorax, 8, 326.

Dillon. J. (1928). Ergebn. med. Strahlenforsch., 3, 289.

Douady, Lardanchet, and Venator (1939). Arch. mid.-chir. Appur. resp., 14, 275 .

Evans, C. J., and Simpson, J. A. (1950). Thorax, 5, 343.

Fatou, Prévost, L., and Prévost, F. (1928). Bull. Soc. míd. Hôp., Paris, 52, 259

Fox, W. (1948). Thorax, 3, 15.

Freedman, B. (1950). Ibid., 5, 169.

French, S. G. (1938). J. rov. nav. med. Serv., $24,349$.

Harley, H. R. S. (1949). Thorax, 4, 1.

Hitzenberger, K. (1927). Das Zwerchfell im gesunden und kranken Zustand, p. 134. Springer, Vienna.

Joannides, M. (1946). Dis. Chest, 12, 89.

Kinzer, R. E.. and Cook, J. C. (1944). Amer. J. Roentgenol., 52, 611. Kirklin, B. R., and Hodgson, J. R. (1947). Ibid., 58, 77.

Königer, H. (1909). Münch. med. Wschr., 56, 282.

Korns, H. M. (1921). Arch. int. Med., 28, 192.

Lerche, W. (1922). Surg. Gynec. Obstet., 34, 224

Macleod, J. G., and Grant, I. W. B. (1954). Thorax, 9, 71

Meyler, L., and Huizinga, E. (1950). J. thorac. Surg., 19, 283.

Miller, H. G., and Stanton, J. B. (1954). Quart. J. Med., n.s. 23, 1. Morison, J. M. W. (1923a). Arch. Radiol. Electrother., 27, 353. (1923b). Ibid., 28, 72.

Pacheco de Figueiredo, J. M., and de Melo, E. M. (1941). Antiseptic, 38, 338.

Perry, K. M. A. (1952). In Marshall, G., and Perry, K. M. A. Diseases of the Chest, vol. 2, p. 375. Butterworth, London.

Quadrone, C. (1912). Riv. crit. Clin. med., 13, 705.

Reed, J. A., and Borden, D. L. (1935). Arch. Surg., Chicago, 31, 30. Richardson, J. S. (1942). Lancet, 1, 618.

Shanks, S. C., and Kerley, P. (1951). A Text-book of X-ray Diagnosis, vol. 2, p. 221 . Lewis, London.

Walton, H. J. (1924). Amer. J. Roentgenol., 11. 420. 